\title{
Renormalization schemes and renormalons
}

\author{
N.V. Krasnikov and A.A. Pivovarov \\ Institute for Nuclear Research of the Russian Academy of Sciences, Moscow 117312
}

\begin{abstract}
We describe some ways how higher order corrections can reveal themselves if integrated over the infrared region. We show that in different renormalization group (RG) schemes and for some observables one has no factorial divergences. We argue that for treating things in the infrared region it is preferable to start with a RG scheme without the infrared Landau pole in the running coupling constant. The uncertainties for the $\tau$ lepton width resulting from accounting for higher order corrections are discussed.
\end{abstract}

With new high order corrections of perturbation theory hardly available anymore in cases like $e^{+} e^{-}$annihilation or $\tau$ lepton width [1] it is tempting to speculate on the general structure of series within perturbation theory (PT) [2, 3, 4. Some attention has been recently paid to possible factorial divergences in PT series for observables that include integration over an infrared region in momentum space [5, 6, 0, 8]. At the level of diagrams within PT it happens to any observable and the factorial divergence due to the simple bubble chain diagrams is predicted. In fact this prediction is not well justified in QCD because of the choice of a particular subset of diagrams and even of the special contributions (nonabelianization [9]). Next terms of PT expansion can cancel these divergences because such terms become large at small momenta and can not be treated as corrections. In QED there is a formal parameter for ordering diagrams within the $1 / N_{f}$ expansion and the statement about the factorial growth of coefficients can be confirmed by direct computation [10] but it is known also that QED within the $1 / N_{f}$ expansion has practically no sense at all and using it as a guide for general structure of QCD is not well grounded.

In this paper we consider some observables that are represented by integrals over the infrared region and give several ways to define them using the freedom of choice of the $\mathrm{RG}$ scheme. The main conclusion we draw is that the results of integration can easily be made well defined without any nonperturbative (in a strictly defined sense) contributions. These results are ambiguous to the same degree as any ordinary PT series, numerically it can be important because in the infrared region the coupling constant becomes large in most of "natural" RG schemes. However it can be made small as well by some particular choice of extrapolation to low momenta.

The paper is organized as follows. First we define our main object as an integral of some PT expansion over infrared region and show that the corresponding expression 
can contain no factorials depending on the integrand. Even with the simplest version of RG analysis including only the leading $\beta$ function and summation of leading log's, the Landau ghost contributions to different orders in $\alpha_{s}$ can cancel each other. This leads us to conclusion that infrared renormalons or factorial growth of coefficients due to some infrared integration has no invariant meaning.

Next we present a number of examples where integrals are well defined and discuss their properties as functions of the boundary value of the coupling $\alpha_{s}(s)$. It is shown that integrals can be analytic or nonanalytic in $\alpha_{s}(s)$ in the vicinity of $\alpha_{s}(s)=0$ depending on a $\beta$ function used. So, extrapolation of PT evolution to low energy domain can be done in different ways and the results vary strongly.

These two parts accomplish our theoretical consideration of the problem with conclusion that nothing definite can be said about the structure of higher order terms on the basis of some subset of diagrams.

Then we go to practical applications and estimate uncertainties of theoretical predictions for the $\tau$ lepton width when different approaches for defining the integration over infrared region are used. This problem has been widely discussed in the literature (as recent references see, e.g. [11, 12, 13]) so we limit ourselves to qualitatively different versions of changing RG schemes only. The basic (or naive) scheme is one where only self-consistent number of terms of the expansion in the coupling constant is kept. Note that adding all terms proportional to RG log's can produce any answer - just the reflection of the Landau singularity. Then we use the $\mathrm{K}$ scheme [14, 15, 16] where there are no corrections to $R(s)$ and the corresponding $\beta$ function in three-loop approximation acquires an infrared fixed point so that the coupling constant can be extrapolated to the origin without any singularity.

Finally we propose a set of schemes that regularize the infrared behavior of the coupling constant in general and allow one to use any reference scheme for high energy domain. All these schemes are legal and perturbatively equivalent at high energies. The uncertainties that come from low energy region are quite essential as our study shows.

Thus the main object of our interest is the expression of the form

$$
F(s)=\frac{1}{s} \int_{0}^{s} \rho(t) \omega(t) d t
$$

where $\rho(t)$ is given by a PT expansion

$$
\rho(t)=\alpha_{s}(t)+\ldots
$$

while $\omega(t)$ is a kinematical factor smooth in the integration region. Expression (1) enters the finite energy sum rules analysis [17]. We do not dwell upon the peculiarities connected with the analytic continuation [18, 19] and consider eq. (11) as a formal expression. Note 
that the formulation with the help of integration along the circle in the complex plane does not help much by itself because RG improved expressions for PT approximations of physical quantities have wrong analytical properties so the spurious singularities give contributions to physical results [20].

We restrict ourselves to the case of $\omega(t)=1$ and compare

$$
F(s)=\frac{1}{s} \int_{0}^{s} \rho(t) d t \quad \text { and } \quad \rho(s)=\alpha_{s}(s)+\ldots
$$

For technical convenience we introduce a new notation for the coupling constant $a(s)=$ $\beta_{0} \alpha_{s}(s)$ so that asymptotically $a(s) \rightarrow\left(\ln \left(s / \Lambda^{2}\right)\right)^{-1}$ when $s \rightarrow \infty$. A RG equation for our rescaled coupling constant $a(s)$ reads

$$
s \frac{d}{d s} a(s)=\beta(a)=-a^{2}\left(1+c a^{2}+c_{1} a^{3}+\ldots\right)
$$

with rescaled values of coefficients $c=64 / 81, c_{1}=3863 / 4374$ for $n_{f}=3$ in the $\overline{\mathrm{MS}}$ renormalization scheme [21].

Taking $\rho(t)=a(t)$ we find the factorial growth of coefficients of $a(s)$ expansion of $F(s)$ that reflects the presence of infrared Landau ghost (or divergence of the formal series for the integrand)

$$
F(s)=\frac{1}{s} \int_{0}^{s} a(t) d t=a(s) \sum_{n=0}^{\infty} a^{n}(s) n !, \quad a(s)>0
$$

where in the leading approximation

$$
a(t)=\frac{a(s)}{1-a(s) \ln (s / t)}=a(s) \sum_{n=0}^{\infty} a^{n}(s) \ln ^{n}(s / t) .
$$

Last equality in eq. (田) is formal and valid only within the convergence circle of the power series $|a(s) \ln (s / t)|<1$, the use of this equality outside the convergence circle leads to all problems of factorial growth. Factorials can be canceled by higher order terms in the integrand $\rho(t)$. Indeed, for $\rho(t)=a(t)+k a^{2}(t)$ we find for a simplified $\beta$ function $\beta(a)=-a^{2}$ with $c=0$ that corresponds to $n_{f} \sim 8$

$$
\frac{1}{s} \int_{0}^{s}\left(a(t)+k a^{2}(t)\right) d t=a(s)+a(s)(1+k) \sum_{n=1}^{\infty} a^{n}(s) n !
$$

If $k=-1$ there are no factorials in the expression of the form (3) and only first term survives. Thus higher order corrections to the integrand can drastically change the situation with factorials. It is natural because factorials come from the region where higher order corrections are not small and comparable (even much larger) than leading contributions.

In general, we find for integrals of the invariant charge given by eq. (4)

$$
\frac{1}{s} \int_{0}^{s} a(t)^{p} d t=\frac{a(s)^{p}}{(p-1) !} \sum_{n=0}^{\infty}(n+p-1) ! a^{n}(s)
$$


and for $\rho(t)=a(t) \sum_{p=0}^{N} \rho_{p} a^{p}(t)$

$$
\begin{gathered}
\frac{1}{s} \int_{0}^{s} \rho(t) d t=a(s) \sum_{p=0}^{N} \frac{\rho_{p}}{p !} \sum_{n=p}^{\infty} a^{n}(s) n ! \\
=a(s) \sum_{n=N}^{\infty} a^{n}(s) n ! \sum_{p=0}^{N} \frac{\rho_{p}}{p !}+a(s) \sum_{p=0}^{N} \frac{\rho_{p}}{p !} \sum_{n=p}^{N-1} a^{n}(s) n ! .
\end{gathered}
$$

The last addendum is a finite sum (an analog of $a(s)$ in eq. (5)) while the coefficient in front of the infinite sum with factorial terms is a linear combination made from the coefficients $\rho_{p}$ of the observable $\rho(t)$. Inclusion of nonvanishing coefficients of higher order terms of the $\beta$ function is straightforward and only leads to extended combinatorics leaving the statement about the factorial growth unchanged if a $\beta$ function keeps to be negatively defined for all $a$. Even if the factorial growth persists (not complete cancelation between contributions from different orders) its coefficient (the strength) is determined by a linear combination of coefficients $\rho_{n}$ coming from all orders. It is a consequence of the fact that at low energy the formal ordering with $a(s)$ is invalid and all orders contribute a comparable amount to the coefficient of factorial. In other words, factorials appear due to RG log's but in higher orders coefficients of these log's contain not only the $\beta$ function coefficients but lower order terms of observables themselves. So the behavior at large orders of PT for the integral can not be fixed without knowing the whole series for the $\rho(t)$ that is hopeless. The finite series for a $\beta$ function in schemes like 't Hooft's one is illusion - it is still a nonperturbative statement about vanishing coefficients in all orders.

Now we go over to examples without the Landau ghost in the infrared region that are free of any problems with integration or factorial growth. The purpose can be achieved with different means. In higher orders a $\beta$ function can have a fixed point in some scheme. This happens for $e^{+} e^{-}$annihilation in third order in $\mathrm{K}$ scheme without corrections. Or one can write down some new $\beta$ functions that are defined at all values of coupling and determine its evolution for all momenta in a smooth way.

We start with the second option and consider the model $\beta$ function [20]

$$
\beta(a)=-\frac{a^{2}}{1+\kappa a^{2}}, \quad \kappa>0
$$

while $\beta^{a s}(a)=-a^{2}+\ldots$ Then the RG equation (2) has a solution

$$
a(z)=\frac{-\ln \frac{z}{\Lambda^{2}}+\sqrt{\ln ^{2} \frac{z}{\Lambda^{2}}+4 \kappa}}{2 \kappa}
$$

and the unphysical pole at $z=\Lambda^{2}$ of the asymptotic solution $a^{a s}(z)=\left(\ln \left(z / \Lambda^{2}\right)\right)^{-1}$ disappears. Thus, the particular way of summing an infinite number of specific perturbative 
terms for the $\beta$ function can cure the Landau pole problem. Let us stress that there are no nonperturbative terms added but the freedom of choosing a renormalization scheme for an infinite series was used instead. This is however beyond the formal framework of perturbation theory where only finite order polynomials in the coupling constant are allowed as expressions for any quantity.

Formula (6) can be considered either as a pure PT result in some particular RG after an infinite resummation or as a sort of Pade approximation of some real $\beta$ function that might include nonperturbative terms as well. The only important point for us here is that the running coupling obeying the $\mathrm{RG}$ equation with such a $\beta$ function has a smooth continuation to the infrared region.

The effective charge given by eq. (7) has a correct asymptotic behavior at $z \rightarrow \infty$ and no singularity in the whole complex plane with a cut along the positive semiaxis if one considers it as a running coupling constant in Euclidean space. These properties make it a good expansion parameter for physical observables that obey the dispersion relation because it has a proper analytic behavior unlike the asymptotic charge with the Landau pole. Note that this scheme does not solve the problem of strong coupling and even fails to bypass this problem when the expansion for an observable is alternate and higher order terms can make it negative at small enough $s$ that can contradict spectrality for observables of the type of cross-sections. Because the expansion parameter becomes large in infrared region the polynomial approximation is invalid in this domain. Here we encounter a particular case of the general situation that the expansion in unphysical parameter $\alpha_{s}$ is incorrect and the proper way of action is to expand one physical quantity through another. Such formulation of PT is physically more justified. The use of K scheme is a particular example of this approach.

Now we turn to the consideration of the structure of expressions of the form (1) with different extrapolation of the running coupling to infrared region. In general, the RG equation for

$$
F(s)=\frac{1}{s} \int_{0}^{s} a(t) d t
$$

has the form

$$
F(a)+\beta(a) F^{\prime}(a)=a
$$

and the solution of this differential equation can be found with quadratures because it is a linear differential equation of the first order. At simple $\beta$ functions like $\beta(a)=-a^{2}$ the solution of the RG equation (an integral curve) fixed by the boundary condition $a(s) \rightarrow 0$ at $s \rightarrow \infty$ can not be continued to the point $s=0$. The integral curve goes only until $s=\Lambda^{2}$ where there is a vertical asymptote. New information is necessary for continuation 
of the solution to the origin. Consider now an example with

$$
\beta(a)=\frac{-a^{2}}{1+2 a}, \quad a>0 .
$$

In this case the interval $(0, \infty)$ in $s$ is mapped uniquely to the interval $(0, \infty)$ in $a$ and the solution can be uniquely continued to the origin. Still a special consideration of small $s$ region is necessary to determine the correct contribution of the solution of the homogeneous equation. We find

$$
F(a)=a+a^{2}+\Delta F(a)
$$

where $\Delta F(a)$ is a solution of the homogeneous equation

$$
\begin{gathered}
F(a)+\beta(a) F^{\prime}(a)=0 \\
\Delta F(a)=F_{0} a^{2} \exp \left(-\frac{1}{a}\right) .
\end{gathered}
$$

Coefficient $F_{0}$ is determined from the infrared region to be $F_{0}=-1$. The result is

$$
F=a+a^{2}-a^{2} \exp \left(-\frac{1}{a}\right) .
$$

This result can be obtained by explicit integration as well. Namely, the RG equation for the effective charge of eq. (19) is given by

$$
\ln \left(s / \Lambda^{2}\right)=\frac{1}{a}-2 \ln a
$$

and $F(s)$ can be rewritten as $\left(a_{0} \equiv a(s)\right)$

$$
\begin{gathered}
F(s) \equiv F\left(a_{0}\right)=e^{-\left(\frac{1}{a_{0}}-2 \ln a_{0}\right)} \int_{a_{0}}^{\infty} a e^{\left(\frac{1}{a}-2 \ln a\right)}\left(\frac{1}{a^{2}}+2 \frac{1}{a}\right) d a \\
=e^{-\left(\frac{1}{a_{0}}-2 \ln a_{0}\right)} \int_{0}^{1 / a_{0}} e^{\xi}(\xi+2) d \xi=a_{0}+a_{0}^{2}\left(1-e^{-\frac{1}{a_{0}}}\right) .
\end{gathered}
$$

The last term gives the "condensate" contribution to $F(s)$ at $a_{0} \rightarrow 0(s \rightarrow \infty)$. Up to logarithmic corrections

$$
F^{\text {cond }}(s) \sim \frac{\Lambda^{2}}{s} .
$$

At small $s$ the effective charge runs as $a(s) \sim \Lambda / \sqrt{s}$ and

$$
F(s) \sim-\frac{2 \Lambda}{\sqrt{s}} .
$$

Note that the $\beta$ function (9) does not regularize the whole physical quantity: higher order terms in the expansion (like $a^{2}$ ) can be nonintegrable. In this particular example it is the case. 
This simple example shows that for a reasonable definition of the evolution in the infrared region factorials are absent in the expansion. The last term cannot be detected if the equation (8) is integrated by series near $a=0$. Indeed, for $F(a)=\sum_{k=1} f_{k} a^{k}$ one has for eq. (8) with $\beta$ function (9)

$$
f_{1}=1, \quad f_{2}=1, \quad f_{k}-(k-3) f_{k-1}=0, \quad k>2 .
$$

Thus, $f_{k}=0, k>2$. Note that the last equation would lead to factorial growth but instead all higher order coefficients vanish.

To study higher orders of PT the Borel transformation is often used. The Borel analysis for this example goes as follows

$$
F(a)=\int_{0}^{\infty} e^{-\frac{\xi}{a}} B(\xi) d \xi
$$

and the Borel image $B(x)$ is

$$
B(x)=1+\theta(x-1)+x \theta(1-x) .
$$

So the PT series with all coefficients known still does not allow to restore the exact answer through Borel summation. A naive Borel image for polynomials from definition (11) behaves as $B(x)=\sum_{k=1} f_{k} x^{k} /(k-1)$ ! that is correct at small $x$ and completely wrong at large $x$ and has nothing to do with the exact one (12). Being rewritten as

$$
B(x)=1+x+(1-x) \theta(x-1)
$$

the result shows that Borel image is singular (nondifferentiable) at $x=1$ though this is not a pole singularity.

The corresponding example with $\beta$ function (6) can be written as follows

$$
F(s)=a+\frac{\kappa}{3} a^{3}=\frac{1}{s} \int_{0}^{s}\left(a-a^{2}+\frac{\kappa}{3} a^{3}\right) d s .
$$

The RG equation of the type (8) can be integrated by series but the homogeneous equation (10) has no solution satisfying the boundary conditions at small $s$ (constraint on the growth). To understand this feature we note that at small $s$ the running coupling behaves like $a(s) \sim \kappa^{-1} \ln (\Lambda / s)$ and after integration the logarithmic behavior survives. As for the solution of the homogeneous equation it contains a factor

$$
\exp \left(-\frac{1}{a}+\kappa a\right)
$$

that is not logarithmic at small $s(a \rightarrow \infty)$ and can not appear in the resulting expression. In contrast to the previous example all powers of the effective coupling are integrable here. The rate of increase is however quite large for higher order terms. 
Now we define a set of schemes that provide a smooth extrapolation to the infrared region that are a direct generalization of the model (9). The $\kappa$ scheme is determined by the $\beta$ function

$$
\beta_{\kappa}(a)=\frac{\beta(a)}{1-\kappa a^{n} \beta(a)}
$$

where $\beta(a)$ is a $\beta$ function in a reference scheme, $\overline{\mathrm{MS}}$ for instance. The form (14) is chosen because of practical convenience only - it requires no more work as corresponding reference scheme and eliminates the Landau pole in infrared region.

The $\beta$ function given by eq. (14) is bounded at large $a$ and eq. (2) has a solution for $a(z)$ that is defined on the whole positive semiaxis and is free of the Landau pole. The absence of singularities (Landau ghost) allows one to use the evolution of coupling till the origin and destroys the mathematical part of the reasoning of ref. [5] about Borel nonsummability and also Parisi's conjecture [22] looks wrong in this particular scheme.

The solution of the type (7) can be rewritten without using $\Lambda$ in a more traditional form through some intermediate energy scale $\mu$ [20]. The parameter $\Lambda$ has no special meaning anymore as a position of the pole of the running coupling. Thus, in the pure PT framework one can eliminate the Landau pole of the coupling using the freedom of the renormalization scheme choice. The smooth coupling however does not require any nonperturbative effects to regularize the integrals in which it appears.

The solution to the RG equation for the invariant charge in the $\kappa$ scheme is simple because it is closely related to the $\overline{\mathrm{MS}}$ running coupling

$$
\ln \left(s / \Lambda^{2}\right)=\Phi(a)-\kappa \frac{a^{n+1}}{n+1}
$$

where

$$
\Phi(a) \sim \int^{a} \frac{d x}{\beta(x)}
$$

and the normalization is chosen in the form

$$
\Phi(a)=\frac{1}{a}-c \ln \left(\frac{1}{a}+c\right)+\int_{0}^{a}\left(\frac{1}{\beta(\xi)}-\frac{1}{\beta_{(2)}(\xi)}\right) d \xi
$$

with $\beta_{(2)}(a)=-a^{2}(1+c a)$. This gives the standard definition of the parameter $\Lambda$ to be $\Lambda_{\overline{\mathrm{MS}}}$. Two charges are connected through

$$
a_{\kappa}=a-\frac{\kappa}{n+1} a^{n+3}+o\left(a^{n+3}\right) .
$$

The exact connection can be found from eq. (15). So, for $n>1$ they coincide for all observables because in practice there are no calculation beyond the third order.

Still if the corresponding expansion of a physical observable is alternative, higher order corrections can make the physical quantity negative in some part of the integration region. In this sense the approach does not give the full regularization. 
Concluding the theoretical part of the paper we state that there are different ways to bypass the problem of factorial growth of coefficients for integrals from physical observables. The simple change of RG scheme within PT in high energy region can allow a smooth extrapolation of the effective charge into low energy domain that makes all integrals well defined. The change of scheme leads to numerical uncertainties in prediction that is a common feature of any PT result. We now consider these uncertainties for predictions of the $\tau$ lepton width or for the parameter $\alpha_{s}\left(m_{\tau}^{2}\right)$ extracted from experimental data on this width. We stress that we limit ourselves only to "qualitatively" different schemes that describe different infrared behavior of an invariant charge. We do not discuss small variation of schemes within the qualitatively equivalent class.

The expression for the $\tau$ lepton width has the form [23]

$$
R_{\tau}=\int_{0}^{m_{\tau}^{2}} \frac{d s}{m_{\tau}^{2}} 2\left(1-\frac{s}{m_{\tau}^{2}}\right)^{2}\left(1+2 \frac{s}{m_{\tau}^{2}}\right) R(s)
$$

with $R(s)$ given by

$$
R(s)=3\left(1+\frac{\alpha_{s}(s)}{\pi}+\ldots\right)
$$

Using the experimental value (as a recent reference, see [11])

$$
R_{\tau}^{e x p}=3.645 \pm 0.024
$$

and writing

$$
R_{\tau}=3\left(1+\frac{\pi}{\beta_{0}} r_{\tau}\right)
$$

we find

$$
r_{\tau}=a\left(\mu^{2}\right)+\ldots \quad \text { and } \quad r_{\tau}^{e x p}=.4838 \pm 0.018
$$

First, for the sake of completeness and for purposes of normalization we compute the naive $\mathrm{RG}$ result that corresponds to keeping only three terms of expansion in $a\left(\mu^{2}\right)$ with explicit $\mathrm{RG}$ logarithms. Having rewritten $R(s)$ as

$$
R(s)=3\left(1+\frac{\pi}{\beta_{0}} r(s)\right)
$$

one gets for properly normalized QCD contribution (we do not discuss any corrections due to electroweak interactions and take this process only for demonstration of theoretical uncertainties stemming from the choice of RG scheme in QCD)

$$
r(s)=a\left(\mu^{2}\right)+a^{2}\left(\mu^{2}\right)\left(k_{1}+L\right)+a^{3}\left(\mu^{2}\right)\left(k_{2}+L\left(c+2 k_{1}\right)+L^{2}\right)
$$

with $k_{1}=0.7288, k_{2}=-2.0314$ 24, 25], $L=\ln \frac{\mu^{2}}{s}$. Introducing normalized moments

$$
r_{N}=(N+1) \int_{0}^{m_{\tau}^{2}} \frac{d s}{m_{\tau}^{2}}\left(\frac{s}{m_{\tau}^{2}}\right)^{N} \rho(a)
$$


we get the well known result

$$
r_{\tau}=2\left(r_{0}-r_{2}+r_{3} / 2\right)
$$

and

$$
(N+1) \int_{0}^{1} x^{N}(-\ln x)^{p} d x=\frac{p !}{(N+1)^{p}}
$$

This gives

$$
r_{N}=a+a^{2}\left(k_{1}+\frac{1}{N+1}\right)+a^{3}\left(k_{2}+\frac{c+2 k_{1}}{N+1}+\frac{2}{(N+1)^{2}}\right) .
$$

Collecting all together we get the result

$$
r_{\tau}=a+\left(k_{1}+\delta_{1}\right) a^{2}+\left(k_{2}+\left(c+2 k_{1}\right) \delta_{1}+\delta_{2}\right) a^{3} \equiv a+k_{1}^{\prime} a^{2}+k_{2}^{\prime} a^{3}
$$

where $\delta_{1}=19 / 12, \delta_{2}=265 / 72$. The equation for determination $\alpha_{s}\left(m_{\tau}^{2}\right)$ reads

$$
r_{\tau}=r_{\tau}^{e x p}=0.4838 \pm 0.018
$$

We have two options here: to solve equation (22) with the theoretical expression for $r_{\tau}$ given by eq. (refresultth) exactly or perturbatively. Exact answer is

$$
a=0.253 \pm 0.006 \quad \text { and } \quad \alpha_{s}\left(m_{\tau}^{2}\right)=0.353 \pm 0.008
$$

Perturbative method gives

$$
\begin{gathered}
a\left(m_{\tau}^{2}\right)=r_{\tau}-k_{1}^{\prime} r_{\tau}^{2}-\left(k_{2}^{\prime}-2\left(k_{1}^{\prime}\right)^{2}\right) r_{\tau}^{3}+O\left(r_{\tau}^{4}\right) \\
=r_{\tau}-\left(k_{1}+\delta_{1}\right) r_{\tau}^{2}-\left(k_{2}+\left(c+2 k_{1}\right) \delta_{1}+\delta_{2}-2\left(k_{1}+\delta_{1}\right)^{2}\right) r_{\tau}^{3}+O\left(r_{\tau}^{4}\right)=0.564 \pm 0.046
\end{gathered}
$$

and

$$
\alpha_{s}\left(m_{\tau}^{2}\right)=\frac{4 \pi}{9} a\left(m_{\tau}^{2}\right)=0.787 \pm 0.063
$$

Both results are purely perturbative and the errors reflect only the experimental error with the theoretical procedure being strictly fixed. We do not consider any options with choice of $\mathrm{RG}$ without introducing any qualitative change. We also do not discuss any keeping additional terms known due to RG (like $\alpha_{s}^{n} l n^{n}\left(\mu^{2} / s\right)$ ).

Our last remark here is to formulate the result in a $\mathrm{RG}$ invariant way in the spirit of ref. [26]. The simplest (not unique) way is to express the integral through the value of integrand taken at the end point. If $r(s)=a+k_{1} a^{2}+k_{2} a^{3}$ then

$$
\frac{1}{s} \int_{0}^{s} r(t) d t=r(s)+r(s)^{2}+(c+2) r(s)^{3}
$$

and for the $\tau$ lepton width we find

$$
r_{\tau}=r(s)+\delta_{1} r(s)^{2}+\left(\delta_{1} c+\delta_{2}\right) r(s)^{3}=r(s)+\frac{19}{12} r(s)^{2}+\left(\frac{19}{12} c+\frac{265}{72}\right) r(s)^{3} .
$$


Eq. (24) corresponds to the spirit of PT in that respect that it gives the direct relation between two measurable quantities. As for numerical values, the exact solution is $r\left(m_{\tau}^{2}\right)=$ 0.270 while the perturbative one is $r^{P T}\left(m_{\tau}^{2}\right)=0.123$. For corresponding couplings we find $\alpha_{s}^{P T}\left(m_{\tau}^{2}\right)=0.164$ and $\alpha_{s}^{\text {exact }}\left(m_{\tau}^{2}\right)=0.359$. So, these two observables are poorly connected by PT relation that means that the operation of integration disturbs the PT expansion for $r(s)$ strongly. From formula (23) and integration rules for log's we deduce that large contributions of infrared region should be suppressed, i.e. only computation of high order moments are allowed as a PT operation. For three orders of coupling constant expansion and for the $N$ th order moment we require that the change of coefficients would be small enough

$$
r_{N}(r)=r+\frac{1}{N+1} r^{2}+\left(\frac{c}{N+1}+\frac{2}{(N+1)^{2}}\right) r^{3} .
$$

This relation (25) does not include any coefficients $k_{i}$ of the expansion of $r$ in unphysical parameter $\alpha_{\overline{\mathrm{MS}}}$ and reflects only the influence of integration. Requiring this influence to be perturbative we get $N>1-2$ for particular values of $r$ and $c$. The estimate depends on the number of RG log's kept for the expansion: the more log's kept in the expansion the larger the low limit for moments remaining perturbatively related to the integrand. This example again shows that the MS coupling constant is artificial (and auxiliary) quantity. And with integration over regions where PT series is invalid the integration measure can not be chosen arbitrary. Of course, numerically this particular computation can be made more appropriate by choosing some intermediate point $s^{*}$ for normalization instead of the end point and by using $r\left(s^{*}\right), s^{*}<m_{\tau}^{2}$ as an expansion parameter for the integral. This method would correspond to one of ref. [27, 28] but for fixed order of moment $s^{*}$ approaches to $\Lambda^{2}$ if higher orders of log's would be kept. Still, such a formulation of the calculation is more physical one because it connects two measurable quantities directly without intermediate things like $\alpha_{M S}$. The validity of PT expansion in this case means that two quantities commensurate and can be a measure for each other. We see that integration through infrared region violates this property unless the weight function is properly chosen. The choice depends also on the order of PT: at higher orders the suppression must be larger. So, the result of integration and the integrand are poorly connected by PT. If we had only higher moments (say, third one) for the width the situation would be different for this particular order of PT.

In fact, the most successful method in this framework would be the Stevenson's approach [29, 30] that requires an independent optimization for the integral as an independent PT quantity. Being extremely flexible it creates the best scheme for every process and every order of PT independently. We did not check however whether this method gives the convergent answer for the problem at hand. 
Next we consider methods that allow to integrate after applying the RG that is beyond the scope of conventional PT.

First, in three-loop approximation in the $\mathrm{K}$ scheme there is a fixed point for the running coupling and consequently there is no Landau pole problem. Namely, in the scheme where there are no corrections to $r(s)$ the running coupling reads

$$
r(s) \equiv a_{K}=a+k_{1} a^{2}+k_{2} a^{3}
$$

while the $\beta$ function in this scheme is

$$
\beta_{K}\left(a_{K}\right)=-a_{K}^{2}\left(1+c a_{K}+\left(c_{1}-c k_{1}-k_{1}^{2}+k_{2}\right) a_{K}^{2}+\ldots\right) \equiv-a_{K}^{2}\left(1+c a_{K}+c_{1}^{\prime} a_{K}^{2}+\ldots\right)
$$

with $c_{1}^{\prime}=-2.2552$. Fixed point is given by the equation

$$
1+c a_{K}+c_{1}^{\prime} a_{K}^{2}=0
$$

with the solution $a_{K}=0.8637$, and $\left(\alpha_{s}\right)_{K}=(4 \pi / 9) a_{K}=1.206$. The relation between coupling in $\mathrm{K}$ and $\overline{\mathrm{MS}}$ schemes is

$$
a_{K}=a+k_{1} a^{2}+k_{2} a^{3}, \quad a=a_{K}-k_{1} a_{K}^{2}-\left(k_{2}-2 k_{1}^{2}\right) a_{K}^{3} .
$$

Then the procedure is as follows. We compute integrals exactly an find

$$
\alpha_{s}\left(m_{\tau}^{2}\right)_{K}=0.380 \pm 0.013
$$

Computation of $\overline{\mathrm{MS}}$ coupling constant gives two answers again

$$
\begin{gathered}
\alpha_{s}\left(m_{\tau}^{2}\right)^{\text {exact }}=0.361 \pm 0.014 \\
a\left(m_{\tau}^{2}\right)^{p o l}=a_{K}\left(m_{\tau}^{2}\right)-k_{1} a_{K}^{2}\left(m_{\tau}^{2}\right)-\left(k_{2}-2 k_{1}^{2}\right) a_{K}^{3}\left(m_{\tau}^{2}\right), \\
\alpha_{s}\left(m_{\tau}^{2}\right)^{p o l}=\frac{4 \pi}{9} a\left(m_{\tau}^{2}\right)^{p o l}=0.392 \pm 0.017 .
\end{gathered}
$$

Note that the difference between them is larger than the uncertainty due to the experimental error and two intervals hardly overlap.

Finally, we give the results in our $\kappa$ scheme analysis that is more general and universally applicable to integration in any basic scheme as well. This is a generalization of the fixed point approach.

The spectral density in $\kappa$ scheme (with $n=0$ ) up to third order is

$$
\begin{aligned}
& \rho(s)=a_{\kappa}+k_{1} a_{\kappa}^{2}+\left(k_{2}+\kappa\right) a_{\kappa}^{3}, \\
& a_{\kappa}=a-\kappa a^{3}, \quad a=a_{\kappa}+\kappa a_{\kappa}^{3} .
\end{aligned}
$$


The expansion of $\beta$ function reads

$$
\beta\left(a_{\kappa}\right)=-a_{\kappa}^{2}\left(1+c a_{\kappa}+\left(c_{1}-\kappa\right) a_{\kappa}^{2}+\ldots\right)
$$

so introducing $\kappa$ is equivalent at high energies to change of $c_{1}$. At low energies however they are different.

Integration for moments can be easily rewritten in term of the charge itself

$$
\begin{gathered}
r_{N}=(N+1) \int_{0}^{m_{\tau}^{2}} \frac{d s}{m_{\tau}^{2}}\left(\frac{s}{m_{\tau}^{2}}\right)^{N} \rho(a) \\
=(N+1) \int_{\infty}^{a_{\kappa}} \exp (N+1)\left(\Phi(\xi)-\kappa \xi-\Phi\left(a_{\kappa}\right)+\kappa a_{\kappa}\right)\left(\frac{1}{\beta(\xi)}-\kappa\right) \rho(\xi) d \xi .
\end{gathered}
$$

Introducing the variable $\zeta=1 / \xi$ we get the practical version

$r_{N}=(N+1) \int_{0}^{a_{\kappa}^{-1}} \exp (N+1)\left(\Phi\left(\zeta^{-1}\right)-\kappa \zeta^{-1}-\Phi\left(a_{\kappa}\right)+\kappa a_{\kappa}\right)\left(\frac{1}{\zeta^{2} \beta\left(\zeta^{-1}\right)}+\kappa \zeta^{2}\right) \rho\left(\zeta^{-1}\right) d \zeta$.

Results depend on $\kappa$. This is the ordinary RG dependence that is strong enough because the accuracy is different at large momenta where we keep only the expansion and at small momenta where the exact formulae have to be used to make integrals finite. The obtained results are given in Table 1 . Here $a_{\kappa}$ is found from integration, $\alpha_{s}^{\text {naive }}$ is found from the naive (to third order) relation between the schemes

$$
\alpha_{s}^{\text {naive }}=\frac{4 \pi}{9}\left(a_{\kappa}+\kappa a_{\kappa}^{3}\right),
$$

while $\alpha_{s}^{\text {exact }}$ is found from exact formulae for RG scheme relations (15,16).

For finding the parameter $a_{\kappa}$ from the integral it is useful to know the derivative of the integral with respect to the boundary value $a_{\kappa}\left(m_{\tau}^{2}\right) \equiv a_{0}$

$$
\frac{d r}{d a_{0}}=-\frac{1}{\beta_{\kappa}\left(a_{0}\right)} 2\left(r_{0}-3 r_{2}+2 r_{3}\right) .
$$

We can find the derivative RG equation for coupling $a_{\kappa}$ describing its dependence on the parameter $\kappa$

$$
\frac{d a_{\kappa}}{d \kappa}=a_{\kappa} \beta_{\kappa}\left(a_{\kappa}\right) .
$$

This is a particular case of RG equations

$$
\frac{d a}{d c_{n}}=-\beta(a) \int_{0}^{a} \frac{x^{n+3} d x}{\beta^{2}(x)}, \quad n \geq 1,
$$

that describe the dependence of the running coupling on coefficients of the $\beta$ function. Note that the dependence on $c$ is fixed by the choice of the parameter $\Lambda$ to be $\Lambda_{\overline{\mathrm{MS}}}$. The 


\begin{tabular}{|c|c|c|c|}
\hline$\kappa$ & $a_{\kappa}$ & $\alpha_{s}^{\text {naive }}$ & $\alpha_{s}^{\text {exact }}$ \\
\hline 1.5 & 0.2425 & $0.368(16)$ & $0.380(18)$ \\
1.6 & 0.2209 & $0.333(09)$ & $0.342(10)$ \\
1.7 & 0.2118 & $0.318(08)$ & $0.326(09)$ \\
1.8 & 0.2068 & $0.311(07)$ & $0.319(08)$ \\
1.9 & 0.2037 & $0.307(07)$ & $0.315(08)$ \\
2.0 & 0.2016 & $0.304(07)$ & $0.313(08)$ \\
2.1 & 0.2003 & 0.303 & 0.312 \\
2.2 & 0.1993 & 0.303 & 0.311 \\
2.3 & 0.1986 & 0.303 & 0.312 \\
2.4 & 0.1982 & 0.303 & 0.312 \\
2.5 & 0.1979 & 0.303 & 0.313 \\
2.6 & 0.1977 & 0.304 & 0.315 \\
2.7 & 0.1975 & 0.305 & 0.316 \\
2.8 & 0.1975 & 0.306 & 0.318 \\
2.9 & 0.1975 & 0.307 & 0.319 \\
3.0 & 0.1975 & 0.308 & 0.321 \\
3.2 & 0.1976 & 0.310 & 0.325 \\
3.4 & 0.1978 & 0.313 & 0.329 \\
3.6 & 0.1980 & 0.315 & 0.334 \\
\hline
\end{tabular}

Table 1: $\kappa$ scheme results 


\begin{tabular}{|c|c|}
\hline$\kappa$ & $a_{\kappa}$ \\
\hline 1.5 & .2058 \\
1.6 & .2049 \\
1.7 & .2039 \\
1.8 & .2030 \\
1.9 & .2021 \\
2.0 & .2012 \\
2.1 & .2003 \\
2.2 & .1994 \\
2.3 & .1986 \\
2.4 & .1978 \\
2.5 & .1969 \\
2.6 & .1961 \\
2.7 & .1953 \\
\hline
\end{tabular}

Table 2: RG dependence of $a_{\kappa}$ on $\kappa$

extraction of $a_{\kappa}$ is done under the assumption of RG invariance of the expression for the $\tau$ lepton width so the only reliable data for $a_{\kappa}$ can be taken from that part of Table 1 where equation (27) is satisfied. This equation can be easily solved analytically (it is a linear equation if $\kappa$ is considered as a dependent function and $a_{\kappa}$ as an independent variable), we have preferred however to solve it numerically in the vicinity of the value $\left(\kappa=2.1, a_{\kappa}=0.2003\right)$. The solution does not match well the pattern of the dependence of the extracted $a_{\kappa}$ presented in Table 1 that means that higher order terms of perturbative expansion for the width are essential. If the theoretical expression for $r_{\tau}$ was exactly independent of the scheme the extracted charge would satisfy eq. (27). The actual solution with the initial condition $\left(\kappa=2.1, a_{\kappa}=0.2003\right)$ which is our best estimate is presented in Table 2. So the correct pattern is around $1.9<\kappa<2.3$ where the prediction is $\alpha_{s}\left(m_{\tau}^{2}\right)=0.312$.

Note that contrary to possible impression the prediction in fixed point scheme (or in $\mathrm{K}$ scheme) is also nonstable. Indeed, it is easy to introduce a set of schemes parameterized with the fixed point value of the invariant charge - the extracted $\alpha_{s}\left(m_{\tau}^{2}\right)$ will depend on the scheme within the set. A $\beta$ function for such a set could have the form

$$
\beta_{f}(a)=\beta(a)(1+\kappa \beta(a))
$$

that introduces a dependence on an external scheme parameter $\kappa$. All schemes of the type (28) have a fixed point with different value of the coupling constant depending on parameter $\kappa$. 
To conclude, we have exploited the renormalization scheme freedom to show that some infinite subsets of diagrams can be even convergent not just summable in some generalized way. Then with lack of any parameter or criterion for choosing a particular set of diagrams the renormalization scheme freedom - extended to partially summed infinite series - can change the conclusion about the pattern of behavior of PT series in high orders dictated by the leading order RG and by the presence of the infrared pole in the running coupling constant. The very existence of renormalons can well be the attribute of a renormalization scheme with the Landau ghost.

In fact, the dependence of the extracted values of the coupling constant for the $\tau$ lepton width on schemes is rather large because the energy scale $\left(m_{\tau}^{2}\right)$ is quite low. So, it might be reasonable to fix the scheme in an arbitrary, and somehow simplest, way and then to parameterize the low momenta region in the integral sense only without detailed description of the behavior of the running coupling from point to point. This can be done in terms of distribution. Adding the localized distribution like $\delta$ function and its derivatives can make the integral well defined [20, 31]. These localized contributions look as nonperturbative terms.

As for phenomenological applications, e.g. [32, 33, 34, 35, 36], there are models where some extrapolation of the running charge into the infrared region is used for practical calculation (normally under the sign of integration). In case of the $\tau$ lepton width the uncertainties are large. Our results show that with an extrapolation chosen one can not freely add the contribution of gluon condensate to take into account nonperturbative effects and "improve" the computation - it must be coordinated with the continuation of the running coupling constant into infrared region. In fact, the fixed gluon condensate corresponds to a fixed continuation - the pattern of principal value looks very attractive in this respect. An analogous situation appears in a toy model considered in ref. [37].

Thus, the integration over an infrared region involves the strong coupling dynamics and is arbitrary to a large extent. The final expression of the results through the coupling constant at the boundary value is misleading - the series is bad defined in PT and requires some additional inputs concerning the behavior of the running coupling constant at small momenta. The operation of integration must be restricted, it remains valid only if the low $s$ are suppressed by the weight function. Then the result of integration is perturbatively connected to the integrand and the procedure can be formulated in a RG invariant way.

\section{Acknowledgments.}

One of us (NVK) is indebted to participants of CERN Renormalon Workshop for very hot discussions that was one of the motivations for writing this paper. 


\section{References}

[1] K.G.Chetyrkin, "New computing techniques in physics research III", eds. K.H.Becks and D.Perret-Gallix (World Scientific, 1993), p.523.

[2] G. 't Hooft, in: The Whys of Subnuclear Physics, Proceedings of the 15th Inter.School on Subnuclear Physics, Erice, Sicily, 1977, edited by A.Zichichi (Plenum Press, New York, 1979), p.943.

[3] F.David, Nucl.Phys. B209(1982)433.

[4] A.H.Mueller, Nucl.Phys. B250(1985)327.

[5] V.I.Zakharov, Nucl.Phys. B385(1992)452.

[6] M.Beneke, Phys.Lett. B307(1993)154; Nucl.Phys. B405(1993)424.

[7] P.Ball, M.Beneke and V.M. Braun, Nucl.Phys. B452(1995)563.

[8] M.Neubert, CERN-TH-7524-94, Feb.1995, hep-ph/9502264.

[9] D.J.Broadhurst and A.G.Grozin, Phys.Rev. D52(1995)4082;

M.Beneke, V.Braun, Phys.Lett. B348(1995)513;

C.N.Lovett-Turner, C.J.Maxwell, Nucl.Phys. B452(1995)188.

[10] R.Coquereaux, Phys.Rev. D23(1981)2276.

[11] G.Altarelli, P.Nason and G.Ridolfi, Z.Phys. C68(1995)257.

[12] A.L.Kataev and V.V.Starshenko, Mod.Phys.Lett. A10(1995)235.

[13] M.Neubert, CERN-TH-95-112, Oct.1995, hep-ph/9509432.

[14] G.Grunberg, Phys.Lett. B221(1980)70; Phys.Rev. D29(1984)2315.

[15] N.V.Krasnikov, Nucl.Phys. B192(1981)497.

[16] A.L.Kataev, N.V.Krasnikov and A.A.Pivovarov, Phys.Lett. 107(1981)115; Nucl.Phys. B198(1982)508.

[17] N.V.Krasnikov, K.G.Chetyrkin and A.N.Tavkhelidze, Phys. Lett. 76B(1978)83;

N.V.Krasnikov and A.A.Pivovarov, Phys.Lett. B112(1982)397;

N.V.Krasnikov, A.A.Pivovarov and N.N.Tavkhelidze, Z.Phys. C19(1983)301.

[18] N.V.Krasnikov and A.A.Pivovarov, Phys.Lett. B116(1982)168. 
[19] A.A.Pivovarov, Z.Phys. C53(1992)461; Nuovo Cim. 105A(1992)813.

[20] N.V.Krasnikov and A.A.Pivovarov, hep-ph/9510207.

[21] O.V.Tarasov, A.A.Vladimirov, A.Yu.Zharkov, Phys.Lett. B93(1980)429.

[22] G.Parisi, Phys.Lett. 76B(1978)65; Nucl.Phys. B150(1979)163.

[23] E.Braaten, Phys.Rev.Lett. 60(1988)1606;

S.Narison and A.Pich, Phys.Lett. B211(1988)183;

E.Braaten, Phys.Rev. D39(1989)1458;

E.Braaten, S.Narison and A.Pich, Nucl.Phys. B373(1992)581.

[24] S.G.Gorishny, A.L.Kataev and S.A.Larin, Phys.Lett. B259(1991)144.

[25] L.R.Surguladze, M.A.Samuel, Phys.Rev.Lett. 66(1991)560, 2416(erratum); Phys.Rev. D44(1991)1602.

[26] A.Dhar and V.Gupta, Phys.Rev. D29(1984)2822.

[27] S.J.Brodsky, G.P.Lepage and P.B.Mackenzie, Phys.Rev. D28(1983)228.

[28] M.Neubert, Phys.Rev. D51(1995)5924.

[29] P.M.Stevenson, Phys.Rev. D23(1981)2916.

[30] A.C.Mattingly and P.M.Stevenson, Phys.Rev. D49(1994)437.

[31] N.V.Krasnikov and A.A.Pivovarov, hep-ph/9512213.

[32] I.I.Bigi, M.A.Shifman, N.G.Uraltsev, A.I.Vainshtein, Phys.Rev. D50(1994)2234.

[33] M.Beneke and V.M.Braun, Nucl.Phys. B426(1994)301.

[34] A.V.Manohar and M.B.Wise, Phys.Lett. B344(1995)407.

[35] B.R.Webber, B339(1994)148;

Yu.L.Dokshitzer and B.R.Webber, Phys.Lett.B352(1995)451.

[36] R.Akhoury and V.I.Zakharov, UM-TH-95-19, Jun 1995, hep-ph/9507253.

[37] A.A. Penin and A.A. Pivovarov, Preprint INR-894-95, Jun 1995. hep-ph/9506370. 